\title{
Modeling and theoretical description of magnetic hybrid materials-bridging from meso- to macro-scales
}

\begin{abstract}
Magnetic gels and elastomers consist of magnetic or magnetizable colloidal particles embedded in an elastic polymeric matrix. Outstanding properties of these materials comprise reversible changes in their mechanical stiffness or magnetostrictive distortions under the influence of external magnetic fields. To understand such types of overall material behavior from a theoretical point of view, it is essential to characterize the substances starting from the discrete colloidal particle level. It turns out that the macroscopic material response depends sensitively on the mesoscopic particle arrangement. We have utilized and developed several theoretical approaches to this end, allowing us both to reproduce experimental observations and to make theoretical predictions. Our hope is that both these paths help to further stimulate the interest in these fascinating materials.
\end{abstract}

Keywords: ferrogels, magnetorheological elastomers, magnetostriction, mediated interactions, nonlinear stress-strain behavior, scale-bridging

\section{Introduction}

The magnetic hybrid materials that we concentrate on in the following are referred to by various different terms, among them ferrogels, magnetosensitive elastomers, and magnetorheological elastomers. They consist of magnetic or magnetizable particles, typically of colloidal size, that are embedded in a permanently crosslinked, elastic, polymeric medium, possibly swollen by a solvent [1-8]. The particles are too large to migrate through their surrounding elastic environment so that their positions remain permanently fixed. For simplicity, we refer to these materials as magnetic gels and elastomers.

\footnotetext{
*Corresponding author: Andreas M. Menzel, Institut für Physik, Otto-von-Guericke-Universität Magdeburg, Universitätsplatz 2, 39106 Magdeburg, Germany; Theoretische Physik II: Weiche Materie, Heinrich-Heine-Universität Düsseldorf, Universitätsstr. 1, 40225 Düsseldorf, Germany, E-mail: a.menzel@ovgu.de

Hartmut Löwen, Theoretische Physik II: Weiche Materie, Heinrich-Heine-Universität Düsseldorf, Universitätsstr. 1, 40225 Düsseldorf, Germany
}

つ Open Access. () 2020 Andreas M. Menzel and Hartmut Löwen, published by De Gruyter. (c) BY-NC-ND This work is licensed under the Creative Commons Attribution-NonCommercial-NoDerivatives 4.0 International License.

https://doi.org/10.1515/psr-2019-0088 
Mainly two different types of practical realizations of magnetic gels and elastomers can be distinguished. In the first ones, the spatial particle configurations are unstructured and basically isotropic [9-14]. The second ones contain anisotropic, frequently chain-like arrangements of the discrete particles [10, 14-17]. These aggregates can be generated by applying strong, homogeneous, external magnetic fields during the production process, while the particles are still free to move. In response to the directed magnetization, the anisotropic aggregates form. When in this state the elastic medium is built around the particles, their positions are locked in and the anisotropic structures and aggregates get stored permanently in the materials.

It is the magnetoelastic coupling arising from the combination of the two components, namely the magnetic or magnetizable particles and the elastic polymeric surrounding medium that leads to the fascinating characteristics of these materials. Two particularly outstanding features are the changes in global shape and in the overall mechanical stiffness that can be induced reversibly, from outside, on demand, and while the materials are used by applying homogeneous external magnetic fields. Magnetically inducing changes in shape is called magnetostriction, while magnetically achieving modifications of the mechanical properties is referred to as magnetorheological effect.

Various theoretical studies were performed on systems containing regular lattice-type and more random spatial arrangements of the particles, as well as anisotropic and chain-like aggregates. These investigations have demonstrated that the positioning of the embedded particles relative to each other qualitatively influences the overall behavior, that is, for instance, whether a sample elongates or contracts along the axis of an applied magnetic field, or whether its mechanical stiffness under a certain type of deformation increases or decreases [18-25]. Accordingly, to understand the macroscopic behavior of these materials, it is of central importance to study their overall properties based on the particle interactions occurring on the mesoscopic scale. This is the major objective of our work.

Following this scope, we first need to develop appropriate tools to describe the interactions between the particles on the mesoscopic level. Especially, this refers to those interactions mediated by the elastic environment, which will be the main subject of Section 2. Afterward, we can link the mesoscopic characteristics to the overall material behavior. In this context, we address magnetostrictive and magnetorheological effects in Section 3. Along similar lines, a statistical approach that we developed is overviewed in Section 4. Still one step further, we identified relations between the particle configuration and the overall nonlinear stress-strain behavior, which is outlined in Section 5. Finally, we include a list of several possible additional extensions of our considerations in Section 6. 


\section{Behavior of particulate inclusions in elastic environments on the mesoscopic scale}

As a starting point, we need to understand how discrete individual particles behave in magnetic gels and elastomers when they are enclosed by the surrounding elastic medium. External magnetic field gradients impose forces on magnetic or magnetizable inclusions, while magnetic fields may impose torques or induce pairwise magnetic interactions between the particles. In all these cases, the particles push against the surrounding elastic medium and distort it. Elastic distortions are usually long-ranged. Therefore, the configurations of the other particles are affected by the changes in the surrounding elastic medium. More precisely, the other inclusions are displaced and rotated. Since the particles are typically much stiffer than the elastic surroundings, they counteract deformations of their own body shape. This additionally influences the state of distortion of the elastic environment, which in turn through the long-ranged character affects the configuration of all other particles. Therefore, the corresponding interactions between the particles mediated by the elastic surroundings significantly and mutually couple the positions and orientations of all particles to each other.

We considered explicitly the situation of a homogeneous, isotropic, infinitely extended and linearly elastic medium in which magnetizable, rigid and spherical particles are embedded under no-slip surface conditions. Within this framework, we derived explicit analytical expressions for the coupled displacements and rotations of all particles when they are subject to imposed forces and torques [26, 27]. The scheme of solution corresponds to an expansion in the inverse distance between the particles, which we have completed up to including the sixth order [28]. Our characterization was further extended to the overdamped dynamics of well-separated discrete particles in viscoelastic environments [29].

Figure 1 shows a set of three magnetizable particles embedded within a soft elastic gel in an experimental system. Rotating a nearly saturating external magnetic field within the plane of the particle configuration and magnetizing the particles accordingly, the magnetic interactions between them are altered. Consequently, the distances between the spherical inclusions change. As demonstrated, corresponding experimentally measured data points agree very well with our theoretical predictions [26]. There was one simultaneous overall fit parameter, the elastic shear modulus of the elastic environment namely. In this way, the suitability of the approach for micromechanical measurements was demonstrated. Together with dynamic extensions [29], our theoretical framework can further facilitate the interpretation of micro- and nanorheological measurements [30].

In a related context, we showed that two nearby particles under strong mutual magnetic attraction can overcome the counteraction of the elastic medium between them and jump into virtual contact [31]. This magnetomechanical collapse is reversible upon decreasing the mutual magnetic attraction. Under oblique or perpendicular 


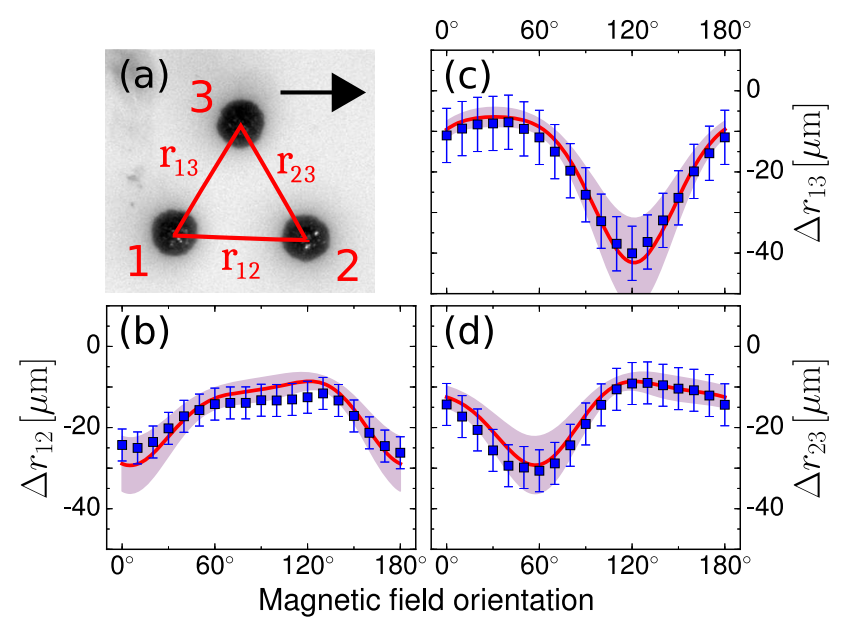

Figure 1: Interplay on the mesoscopic scale between the magnetic interactions of discrete particles and the resulting deformations of a surrounding elastic environment. (a) Far away from any boundary, three magnetizable nickel particles of diameters $208.5 \pm 2.3 \mu \mathrm{m}$ were embedded in a very soft elastic gel. An external magnetic field that magnetizes the particles was rotated clockwise in the particle plane, starting from the orientation of the black arrow. (b)-(d) Changes in the distances between the particles result from the modified magnetic interactions because of the field rotation. Data points and error bars represent the experimental measurements, the (red) lines follow from our theory with the shaded areas marking the associated uncertainty regime. From fitting the theory to the experimental data points, the shear modulus of the elastic gel was determined to be $76.3 \pm 11.7 \mathrm{~Pa}$. Reprinted figure with permission from Ref. [26]. Copyright 2016 by the American Physical Society (DOI:10.1103/ PhysRevLett.117.238003).

magnetic fields, longer linear chains of magnetic particles in very soft magnetic gels showed a characteristic buckling type of distortion with multiple buckles along their contour [32]. Apart from that, in extension of a previous work on the stochastic motion of a particle through an extremely shear-thinning environment [33], we characterized the stochastic motion through shear-thickening surroundings while additionally driven, for instance, by a constant magnetic field gradient [34 $]^{1}$. Beyond the passive motion of individual spherical particles, we analyzed the diffusive behavior of more irregularly-shaped passive objects in fluid environments [35], and the consequences of additional self-propulsion in clusters of magnetic dipolar particles [36]. These considerations will be important when in the future the driven or active transport near or through biological membranes [37-39] will be investigated more intensely, for instance motivated by the use of magnetic particles as drug carriers. The distortions arising from thermophoretic effects while heating a particle in a surrounding elastic gel were analyzed as well [40], an aspect that might be relevant during hyperthermic cancer

1 In both studies [33, 34] the stochastic driving forces are rather thought to be imposed from outside with constant absolute value of their averaged squared amplitude than of thermal origin. 
treatment. Finally, we also studied to lowest order the interactions mediated between discrete particles by a surrounding elastic medium nearby a rigid either free-slip or noslip surface bounding the enclosing elastic environment [41].

\section{From the mesoscopic particle configuration to overall magnetostrictive and magnetorheological effects}

Next, to bridge from the mesoscopic positioning of the particles relatively to each other to the overall, possibly macroscopic behavior of the whole system, we concentrated on two different effects characteristic for magnetic gels and elastomers. First, these are magnetostrictive effects, that is, overall distortions when the systems are magnetized by a homogeneous external magnetic field. Second, we refer to magnetorheological effects, implying as a function of the applied magnetic field reversible changes in the global mechanical stiffness.

Concerning our investigations on magnetostriction, we followed the same theoretical path as outlined in Section 2. However, instead of considering an infinitely extended system, we now assumed the particles to be embedded in a linearly elastic finite-sized sphere. When the particles are magnetized, leading to each particle carrying for simplicity an identical dipole moment, they distort the surrounding elastic material through the resulting pairwise magnetic attraction or repulsion between them. We additionally included the interactions between the particles mediated by the distortions of their elastic environment to lowest order. Superimposing the contributions of all magnetized inclusions, we calculated the overall deformation of the system [42]. The underlying mathematical expressions are analytical and therefore contain the basically infinite amount of degrees of freedom involved in the distortion of the elastic sphere.

It turned out that the nature of the global deformation is strongly connected to the internal particle arrangement. For example, we evaluated simple cubic, body- and face-centered cubic, and rectangular regular lattice configurations of about one thousand particles with different homogeneous orientations of the magnetization. Whether the sphere elongates or contracts along the magnetization direction depends significantly on the mutual particle positioning, on the orientation of the magnetization axis, and on the value of the Poisson ratio quantifying the compressibility of the elastic material [42]. For randomized particle configurations, we found a tendency of elongation along the magnetization, in agreement with corresponding experimental observations [11, 43].

In order to address the magnetorheological effect, that is, resulting changes in the overall mechanical stiffness upon changes in the magnetization, we resorted to reduced dipole-spring models [44-49]. Each particle is represented by a sphere that 
again carries the same magnetic dipole moment at its center. To generate an elastic network, the spheres are linked by elastic springs. In this way, the complex elastic composite material is mapped to a discretized magnetoelastic network. The procedure allows to effectively numerically determine equilibrium ground states for different magnitudes of magnetization. For each ground state, the different mechanical moduli quantifying the stress necessary to achieve a certain type of deformation can be calculated in the framework of linear response.

It turned out that whether under weak induced magnetic interactions the mechanical moduli increase or decrease strongly depends on the particle configuration and on the orientation of the magnetization direction. We evaluated various different regular lattices and also randomized particle configurations [45, 47]. Also particle arrangements extracted from real experimental samples by X-ray tomographic means were evaluated [45, 46, 48]. Upon strong magnetization, we found an internal restructuring to set in. During this process, against the elastic restoring forces of the springs, particles collapse toward each other into virtual contact and form chain-like aggregates. We found this effect to be accompanied by a significant increase in the mechanical stiffness, see Figure 2, in qualitative agreement with corresponding experimental observations [13]. The dynamic moduli, quantifying the storage and loss parts of the dynamic response of the systems, were evaluated as a function of the magnetization and for different particle arrangements as well $[47,48]$.

(a)

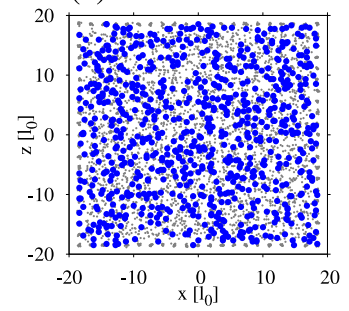

(b)

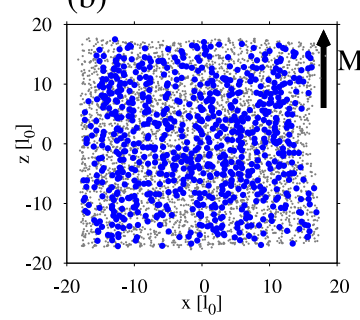

(c)

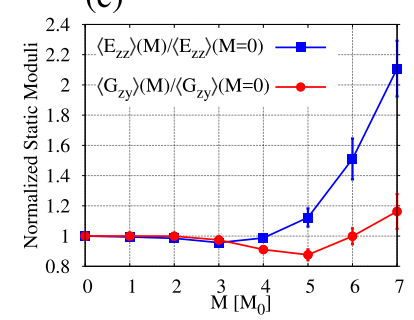

Figure 2: Dipole-spring approach to magnetic gels or elastomers describing the associated magnetorheological effect. (a) Corresponding side view, with the darker (blue) dots indicating magnetizable particles, the positions and volumes of which were extracted from a real experimental sample. Lighter (gray) dots mark additional interstitial network points of the elastic mesh of springs. The springs are not indicated for clarity. Here $I_{0}$ is a typical length scale set by the particle size. (b) When the system is strongly magnetized, by eye one can identify anisotropic structures arising along the axis of magnetization $M$. (c) We averaged the moduli $E_{z z}$, associated with elongations or contractions along the magnetization direction, and $G_{z y}$, connected to shears with $M$ in the shear plane but displacements perpendicular to $M$, over several different numerical realizations of the elastic mesh of springs between the particles. For weak (homogeneous) magnetizations, the moduli tend to slightly decrease. They strongly increase when the magnetically-induced internal restructuring becomes significant. $M_{O}$ sets the unit of magnetization. The system in (b) was magnetized to $|\mathrm{M}|=7 M_{O}$. Reproduced from Ref. [48]. (C IOP Publishing. Reproduced with permission. All rights reserved (DOI:10.1088/1361-648X/aaaeaa). 
As an extension, we evaluated the overdamped particle dynamics using dipolespring systems [46, 49]. First, in qualitative agreement with corresponding experimental observations [50,51], an increase of the eigenfrequencies of the systems was found for increasing magnetization [46]. However, second, it came as a bit of a surprise that, in spite of the overdamped nature, we could identify a shock-wave type of dynamics when a straight chain-like system contracts or elongates after switching on or off strong magnetic attractions between the particles [49]. The wave fronts separate high- and low-density regions.

Apart from that, it turned out that the investigated dipole-spring models prove similarly useful in the characterization of other types of complex elastic composite materials, particularly electrorheological elastomers [52, 53]. In the future, they may also play a certain role in explicitly linking mesoscopic theoretical characterizations to macroscopic theories [54-58]. To provide such a link in a first step [59], we have calculated from a simple homogeneous mesoscopic model picture the material coefficients of the static part of a macroscopic hydrodynamic theory [60] for anisotropic magnetic gels. In this way, we confirmed and illustrated the nature of the terms in the macroscopic approach and provided explicit expressions of the associated material parameters as functions of the mesoscopic properties.

\section{Statistical particle-scale characterization and overall material properties}

Another way to link the properties of the system from the level of the individual particles to the overall material behavior is given by statistical theories. To this end, we developed a classical density functional theory to describe the magnetorheological effect of a system of elastically linked particles. The statistical approach naturally includes thermal fluctuations in the individual particle positions.

In principle, density functional theory is exact in characterizing equilibrium systems [61, 62]. When solving for the equilibrium state, a particle-scale profile for the noise-averaged density distribution is obtained, related to the probability of finding a particle at a certain position in space. Nevertheless, approximations are often needed in reality to handle the mutual interactions between the particles.

To be able to apply the framework of classical density functional theory to elastic composite materials, we first had to clarify a central conceptual issue [63, 64]. Classical density functional theory was developed to describe liquid states. In the statistical characterizations of monodisperse liquids, the particles can effectively be treated as identical or indistinguishable, the latter at least from an averaging perspective. The situation is genuinely different for a given sample of an elastic composite material like a magnetic gel or elastomer. There, the colloidal particles are trapped by the surrounding elastic matrix and their positions relative to each other are fixed permanently. 
This renders the particles distinguishable. From another perspective, when mapping the situation to simple dipole-spring networks, see Section 3, the elastic springs between the particles are permanent and neither cut nor reconnected over time. Therefore, each particle is permanently linked to a given set of specific other particles and solely elastically interacts with this subset. Consequently, again we can distinguish the particles by their relative positioning within this elastic interaction network. The solution was to map these discrete elastic spring interactions that distinguish between individual particles to effective potential interactions that do not distinguish between individual particles but lead to the same appearance of the system [63, 64].

So far, we have considered one-dimensional [63] and two-dimensional [64] particle arrangements using effective potential interactions. Changes in the system behavior upon magnetization were evaluated for attractive and repulsive magnetic particle interactions, respectively. We evaluated the associated density profiles and the magnetorheological effect, that is, the changes in the elastic moduli of the overall systems upon magnetization. For moderate amplitudes of magnetization, we found a decrease in the compressive elastic modulus in the one-dimensional configuration under mutual magnetic attraction of the particles [63]. Vice versa, the compressive and shear elastic moduli typically increased under mutual magnetic repulsion in the two-dimensional arrangements [64]. An exception are dense two-dimensional configurations, in which the magnetic repulsion can add to evade hard steric interactions between the particles. Moreover, as expected, we observed a positive magnetostrictive effect for the overall planar system under internal magnetic repulsion, that is, an increase in the system size [64]. For the one-dimensional configurations, we accounted for an additional embedding in a surrounding elastic environment by introducing an additional external anchoring potential [63], leading to the observations depicted in Figure 3.

All of our results were tested against Monte-Carlo simulations based on the effective potentials mentioned above and on genuine elastic spring interactions [63, 64]. We found good agreement in the parameter regimes that we concentrated on, see Figure 3. The extension from one- to two-dimensional configurations significantly reduced peculiar fluctuation effects that appeared in the one-dimensional arrangement. Further extensions to three-dimensional configurations will certainly allow for a more explicit comparison with actual experimental observations, possibly by using experimentally measured pair distribution functions for the particle arrangement as an input [65].

\section{Linking the particle scale to the overall nonlinear stress-strain behavior}

To establish a bridge from the particulate structures in magnetic gels and elastomers to their overall nonlinear stress-strain behavior, we turned to corresponding simulation 
(a)

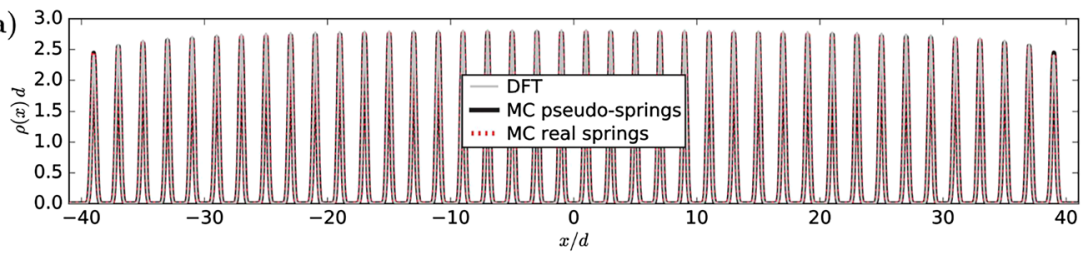

(b)

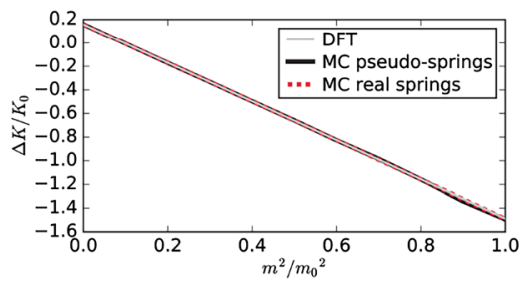

Figure 3: Statistical approach to magnetic gels and elastomers and to the magnetorheological effect via density functional theory, here for a one-dimensional model system of particles embedded in an elastic environment. (a) The density profile $\rho(x)$ of the magnetic particles identifies their location, with $d$ the particle diameter. Since thermal fluctuations are included, the individual peaks have finite width. We first mapped the situation to a dipole-spring system ("real springs"), while the springs were then replaced by effective potential interactions ("pseudo-springs"). Monte-Carlo simulations ("MC") confirm the results of the density functional theory ("DFT"). (b) Rescaled changes $\Delta K / K_{0}$ of the compressive elastic modulus of the one-dimensional particle configuration as a function of increasing rescaled quadratic magnitude of the magnetic moments $\left(\mathrm{m} / \mathrm{m}_{0}\right)^{2}$ of the individual particles. The attractive magnetic particle interactions here lead to a decrease in the elastic modulus. Reproduced from Ref. [63]. (C) IOP Publishing. Reproduced with permission. All rights reserved (DOI:10.1088/1361-648X/aa73bd).

approaches [66, 67]. For this purpose, we considered rigid, finite-sized, spherical magnetic particles that carry permanent magnetic dipoles at their centers. These particles form chain-like aggregates with interparticle gaps of finite distance and are embedded under no-slip surface conditions into an elastic environment. The latter was discretized into a tetrahedral mesh, the elastic distortions of each tetrahedron being quantified by a nearly-incompressible nonlinear elastic deformation energy of the NeoHookean type ${ }^{2}$. Resulting systems contained several mutually shifted chain-like aggregates; see Figure 4(a) for a typical example system. We numerically clamped the systems at their faces where the chains start and end. Then we stretched the systems by small successive increments, allowing for an equilibration in each step of additional elongation. During this procedure, we recorded the overall forces necessary to achieve a certain amount of stretching. Figure 4(b) shows a resulting nonlinear force-strain curve averaged over 20 realizations of the systems.

2 There is a misplaced bracket in the expression for the elastic deformation energy density corresponding to the nearly-incompressible Neo-Hookean hyperelastic model as listed in Eq. (S1) of the supplementary material of Ref. [66] and in Eq. (1) of Ref. [67]. The first term should read $\left(\mu / 2 \operatorname{Tr}\left\{F^{t} \cdot F\right\}-3\right)$ [instead of $\left.\mu / 2\left(\operatorname{Tr}\left\{\mathrm{F}^{t} \cdot \mathrm{F}\right\}-3\right)\right]$. This is a pure typo and has been treated correctly in the simulations. 
(a)

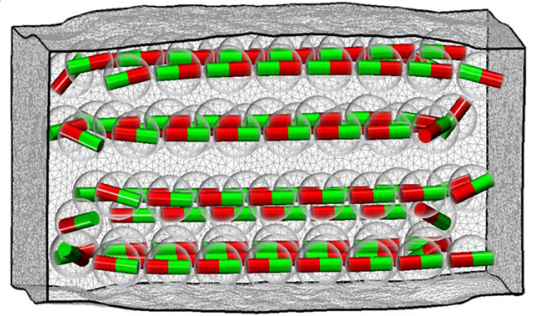

(b)

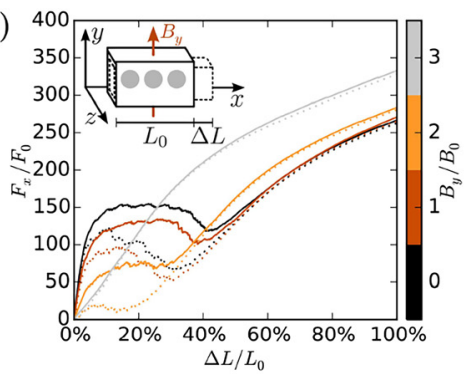

Figure 4: Nonlinear stress-strain behavior of numerical systems representing anisotropic magnetic gels or elastomers that contain chain-like particle aggregates. (a) Here, the particles carry permanent dipole moments indicated by the bar magnets that can freely rotate relatively to the particle frames. The surrounding elastic medium embeds the particles under no-slip surface conditions and is discretized into an elastic tetrahedral mesh. (b) Numerically clamping the systems at their faces, stretching them along the chain axes, and plotting the necessary rescaled stretching force $F_{x} / F_{0}$ averaged over several numerical realizations as a function of elongation $\Delta L / L_{0}$, pronounced nonlinear behavior emerges. This behavior results from the intrinsic coupling between magnetic and elastic properties. Applying a perpendicular magnetic field of rescaled magnitude $B_{y} / B_{0}$, the pronounced intermediate nonlinearity can be altered and even be removed approximately. Reprinted from Ref. [66], with the permission of AIP Publishing (https://doi.org/10.1063/1.4934698).

Our results reveal a pronounced nonlinear stress-strain behavior [66, 67], see the black curve in Figure 4(b). This behavior emerges from the interplay between the nonlinear magnetic particle interactions and the elastic properties of the surrounding medium. At low magnitudes of imposed strain, the magnetic particles are close to each other along the chains and any stretching needs to work against the resulting strong magnetic attraction. This leads to the initial steep increase of the curve. At a certain threshold and at the weakest point of all chains, the stretching force is strong enough to increase the gap between two particles and to match the magnetic attraction in magnitude. Once this barrier is mastered, the gap between the particles suddenly increases by a larger amount, because the magnetic attraction decreases with the further increasing distance between the particles. This leads to a drop of the force-strain curve. In fact the plateau emerging at intermediate strains in Figure 4(b) represents many successive such drops resulting from corresponding events throughout the system, until all chains are disintegrated.

Moreover, in the situation considered in Figure 4, the magnetic dipole of each particle is free to reorient relatively to the particle frame. Thus, when particles are separated from each other along the stretching axis, but lateral particles approach each other, the magnetic moments will flip to minimize the overall magnetic interaction energy. This effect additionally contributes to the appearance of the curves and to a big extent causes the trough that follows the plateau in Figure 4(b). Finally, when all aggregates are disintegrated at large strains, mainly the elastic medium is stretched further, which leads to a comparatively regular behavior. 
From these considerations, it also becomes plausible that the nonlinear stress-strain behavior can be tuned by external magnetic fields, as for the colored curves in Figure 4(b). Strong perpendicular magnetic fields can even switch off the nonlinearity by inducing a perpendicular orientation of the magnetic moments from the start, see the light curve in Figure 4(b).

In addition to that, we also analyzed the situation for permanent magnetic dipole moments being anchored to the particle frames [67]. All magnetic moments initially point into the same direction along the chain axes, or they point into opposite directions for different chains. Overall, the effects resulting from the stretching and disintegration of the chains against their internal magnetic cohesion are still at work, in contrast to their unhindered flipping of magnetic moments.

From a more macroscopic point of view, the overall nonlinear response is reminiscent of the nonlinear stress-strain behavior of nematic liquid-crystalline monodomain elastomers $[68,69]$. There, likewise, reorientation affects couple to elastic deformations and lead to the overall nonlinear stress-strain response of the systems.

\section{Conclusions}

In summary, we utilized and developed various theoretical tools and approaches to characterize the behavior of magnetic gels and elastomers on the mesoscopic scale of the individual magnetic or magnetizable particles embedded in the permanent elastic polymeric environment, and to connect the corresponding properties to the overall system behavior. Among our approaches were analytical calculations within the framework of linear elasticity theory, discretized dipole-spring models, classical density functional theory, and simulations of the nonlinear system behavior. We concentrated on the evaluation of interactions between the particles mediated by the elastic environment, resulting overall magnetostrictive and magnetorheological effects, that is, magnetically induced overall distortions and changes in the overall mechanical features of the systems, respectively, as well as overall nonlinear stressstrain properties. Several new types of behavior were revealed or quantified in this way.

Nevertheless, our work opens the way for several extensions in the future. On the particle scale, our analytical descriptions can be widened to characterize the interactions mediated by the elastic environment between elongated particles [70]. Furthermore, quantifying particle approaches from finite separation to very close locations still represent a major challenge [31, 71]. Similarly, extensions to maintain the volume of the overall system in the dipole-spring approaches under significant internal restructuring [47, 48] are desirable. Concerning our density functional theories [63, 64], the step towards three-dimensional particle configurations represents a natural next level. Eventually, so far our investigations on the nonlinear stress-strain behavior [66, 67] form a theoretical prediction, and we would be fascinated by corresponding experimental realizations. 
Overall, we concentrated on characterizations on the mesoscopic scale and connections to the macroscopic material behavior. However, substantiating the mesoscopic models by scale connections [72] from still more microscopic analyses [73, 74] represents another important direction. Some of the methods developed in the theoretical characterization of magnetic gels and elastomers may also be helpful to investigate related problems in other types of elastic materials, for instance transport through possibly charged polymeric pores [75] or changes in adhesive properties through modified elastic moduli [76]. Finally, we point to another field of growing current interest concerning colloidal composite systems, namely active microswimmers in suspension [3, 77]. Many aspects of the descriptions developed in the present context will also be useful to study details within this related field, particularly when magnetically steerable elastic composite microswimmers are addressed [78].

Acknowledgment: We acknowledge the contributions of many students and postdocs with whom we had the pleasure to collaborate within this topical context and who contributed to our works in this field over the past few years. Among them are Elshad Allahyarov, Sonja Babel, Peet Cremer, Abdallah Daddi-Moussa-Ider, Lukas Fischer, Segun Goh, Borge ten Hagen, Andreas Kaiser, Giorgio Pessot, Mate Puljiz, and Mitsusuke Tarama. Moreover, we thank the German Research Foundation (DFG) for continuous support over the past few years via the SPP 1681, Günter K. Auernhammer, Dmitry Y. Borin, Thomas Gundermann, Christian Holm, Shilin Huang, Karl A. Kalina, Markus Kästner, Johannes Nowak, Stefan Odenbach, Christopher Passow, Malte Schümann, Joachim Wagner, and Rudolf Weeber for the fruitful collaborations within the SPP 1681, as well as Stefan Odenbach for the substantial and ongoing effort in establishing and coordinating this stimulating program.

Author contribution: All the authors have accepted responsibility for the entire content of this submitted manuscript and approved submission.

Research funding: German Research Foundation (DFG) through the SPP 1681, grant nos. ME 3571/3 and LO 418/16.

Conflict of interest statement: The authors declare no conflicts of interest regarding this article.

\section{References}

1. Filipcsei G, Csetneki I, Szilágyi A, Zrínyi M. Magnetic field-responsive smart polymer composites. Adv Polym Sci 2007;206:137-89.

2. Ilg P. Stimuli-responsive hydrogels cross-linked by magnetic nanoparticles. Soft Matter 2013;9: 3465-8.

3. Menzel AM. Tuned, driven, and active soft matter. Phys Rep 2015;554:1-45.

4. Odenbach S. Microstructure and rheology of magnetic hybrid materials. Arch Appl Mech 2016;86: 269-79. 
5. Lopez-Lopez MT, Durán JDG, Iskakova LY, Zubarev AY. Mechanics of magnetopolymer composites: a review. J Nanofluids 2016;5:479-95.

6. Weeber R, Hermes M, Schmidt AM, Holm C. Polymer architecture of magnetic gels: a review. J Phys Condens Matter 2018;30:063002.

7. Menzel AM. Mesoscopic characterization of magnetoelastic hybrid materials: magnetic gels and elastomers, their particle-scale description, and scale-bridging links. Arch Appl Mech 2019;89:17-45.

8. Weeber R, Kreissl P, Holm C. Studying the field-controlled change of shape and elasticity of magnetic gels using particle-based simulations. Arch Appl Mech 2019;89:3-16.

9. Zrínyi M, Barsi L, Büki A. Deformation of ferrogels induced by nonuniform magnetic fields. J Chem Phys 1996;104:8750-6.

10. Jolly MR, Carlson JD, Muñoz BC. A model of the behaviour of magnetorheological materials. Smart Mater Struct 1996;5:607-14.

11. Gollwitzer C, Turanov A, Krekhova M, Lattermann G, Rehberg I, Richter R. Measuring the deformation of a ferrogel sphere in a homogeneous magnetic field. J Chem Phys 2008;128: 164709.

12. Evans BA, Fiser BL, Prins WJ, Rapp DJ, Shields AR, Glass DR, et al. A highly tunable silicone-based magnetic elastomer with nanoscale homogeneity. J Magn Magn Mater 2012;324:501-7.

13. Schümann M, Odenbach S. In-situ observation of the particle microstructure of magnetorheological elastomers in presence of mechanical strain and magnetic fields. J Magn Magn Mater 2017;441:88-92.

14. Schümann M, Gundermann T, Odenbach S. Microscopic investigation of the reasons for fielddependent changes in the properties of magnetic hybrid materials using $\mathrm{X}$-ray microtomography. Arch Appl Mech 2019;89:77-89.

15. Collin D, Auernhammer GK, Gavat O, Martinoty P, Brand HR. Frozen-in magnetic order in uniaxial magnetic gels: preparation and physical properties. Macromol Rapid Commun 2003;24:737-41.

16. Günther D, Borin DY, Günther S, Odenbach S. X-ray micro-tomographic characterization of fieldstructured magnetorheological elastomers. Smart Mater Struct 2011;21:015005.

17. An H-N, Groenewold J, Picken SJ, Mendes E. Conformational changes of a single magnetic particle string within gels. Soft Matter 2014;10:997-1005.

18. Wood DS, Camp PJ. Modeling the properties of ferrogels in uniform magnetic fields. Phys Rev E 2011;83:011402.

19. Ivaneyko D, Toshchevikov VP, Saphiannikova M, Heinrich G. Magneto-sensitive elastomers in a homogeneous magnetic field: a regular rectangular lattice model. Macromol Theory Simul 2011; 20:411-24.

20. Stolbov OV, Raikher YL, Balasoiu M. Modelling of magnetodipolar striction in soft magnetic elastomers. Soft Matter 2011;7:8484-7.

21. Gong X, Liao G, Xuan S. Full-field deformation of magnetorheological elastomer under uniform magnetic field. Appl Phys Lett 2012;100:211909.

22. Han Y, Hong W, Faidley LE. Field-stiffening effect of magneto-rheological elastomers. Int J Solid Struct 2013;50:2281-8.

23. Zubarev AY. Effect of chain-like aggregates on ferrogel magnetodeformation. Soft Matter 2013;9: 4985-92.

24. Metsch P, Kalina KA, Spieler C, Kästner M. A numerical study on magnetostrictive phenomena in magnetorheological elastomers. Comput Mater Sci 2016;124:364-74.

25. Romeis D, Toshchevikov V, Saphiannikova M. Effects of local rearrangement of magnetic particles on deformation in magneto-sensitive elastomers. Soft Matter 2019;15:3552-64.

26. Puljiz M, Huang S, Auernhammer GK, Menzel AM. Forces on rigid inclusions in elastic media and resulting matrix-mediated interactions. Phys Rev Lett 2016;117:238003. 
27. Puljiz M, Menzel AM. Forces and torques on rigid inclusions in an elastic environment: resulting matrix-mediated interactions, displacements, and rotations. Phys Rev E 2017;95: 053002.

28. Puljiz M, Menzel AM. Displacement field around a rigid sphere in a compressible elastic environment, corresponding higher-order Faxén relations, as well as higher-order displaceability and rotateability matrices. Phys Rev E 2019;99:053002.

29. Puljiz M, Menzel AM. Memory-based mediated interactions between rigid particulate inclusions in viscoelastic environments. Phys Rev E 2019;99:012601.

30. Remmer H, Roeben E, Schmidt AM, Schilling M, Ludwig F. Dynamics of magnetic nanoparticles in viscoelastic media. J Magn Magn Mater 2017;427:331-5.

31. Puljiz M, Huang S, Kalina KA, Nowak J, Odenbach S, Kästner M, et al. Reversible magnetomechanical collapse: virtual touching and detachment of rigid inclusions in a soft elastic matrix. Soft Matter 2018;14:6809-21.

32. Huang S, Pessot G, Cremer P, Weeber R, Holm C, Nowak J, et al. Buckling of paramagnetic chains in soft gels. Soft Matter 2016;12:228-37.

33. Menzel AM, Goldenfeld N. Effect of Coulombic friction on spatial displacement statistics. Phys Rev E 2011;84:011122.

34. Menzel AM. Velocity and displacement statistics in a stochastic model of nonlinear friction showing bounded particle speed. Phys Rev E 2015;92:052302.

35. Passow C, Ten Hagen B, Löwen H, Wagner J. Depolarized light scattering from prolate anisotropic particles: the influence of the particle shape on the field autocorrelation function. J Chem Phys 2015;143:044903.

36. Kaiser A, Popowa K, Löwen H. Active dipole clusters: from helical motion to fission. Phys Rev $\mathrm{E}$ 2015;92:012301.

37. Daddi-Moussa-Ider A, Goh S, Liebchen B, Hoell C, Mathijssen AJTM, Guzmán-Lastra F, et al. Membrane penetration and trapping of an active particle. J Chem Phys 2019;150:064906.

38. Daddi-Moussa-Ider A, Kaoui B, Löwen H. Axisymmetric flow due to a stokeslet near a finite-sized elastic membrane. J Phys Soc Jpn 2019;88:054401.

39. Daddi-Moussa-Ider A, Liebchen B, Menzel AM, Löwen $\mathrm{H}$. Theory of active particle penetration through a planar elastic membrane. New J Phys 2019;21:083014.

40. Puljiz M, Orlishausen M, Köhler W, Menzel AM. Thermophoretically induced large-scale deformations around microscopic heat centers. J Chem Phys 2016;144:184903.

41. Menzel AM. Force-induced elastic matrix-mediated interactions in the presence of a rigid wall. Soft Matter 2017;13:3373-84.

42. Fischer L, Menzel AM. Magnetostriction in magnetic gels and elastomers as a function of the internal structure and particle distribution. J Chem Phys 2019;151:114906.

43. Filipcsei G, Zrínyi M. Magnetodeformation effects and the swelling of ferrogels in a uniform magnetic field. J Phys Condens Matter 2010;22:276001.

44. Annunziata MA, Menzel AM, Löwen H. Hardening transition in a one-dimensional model for ferrogels. J Chem Phys 2013;138:204906.

45. Pessot G, Cremer P, Borin DY, Odenbach S, Löwen H, Menzel AM. Structural control of elastic moduli in ferrogels and the importance of non-affine deformations. J Chem Phys 2014;141: 015005.

46. Tarama M, Cremer P, Borin DY, Odenbach S, Löwen H, Menzel AM. Tunable dynamic response of magnetic gels: impact of structural properties and magnetic fields. Phys Rev E 2014;90:042311.

47. Pessot G, Löwen H, Menzel AM. Dynamic elastic moduli in magnetic gels: normal modes and linear response. J Chem Phys 2016;145:104904. 
48. Pessot G, Schümann M, Gundermann T, Odenbach S, Löwen H, Menzel AM. Tunable dynamic moduli of magnetic elastomers: from characterization by $\mathrm{X}$-ray micro-computed tomography to mesoscopic modeling. J Phys Condens Matter 2018;30:125101.

49. Goh S, Menzel AM, Löwen H. Dynamics in a one-dimensional ferrogel model: relaxation, pairing, shock-wave propagation. Phys Chem Chem Phys 2018;20:15037-51.

50. Becker TI, Raikher YL, Stolbov OV, Böhm V, Zimmermann K. Dynamic properties of magnetosensitive elastomer cantilevers as adaptive sensor elements. Smart Mater Struct 2017;26:095035.

51. Becker TI, Zimmermann K, Borin DY, Stepanov GV, Storozhenko PA. Dynamic response of a sensor element made of magnetic hybrid elastomer with controllable properties. J Magn Magn Mater 2018;449:77-82.

52. Allahyarov E, Löwen H, Zhu L. A simulation study of the electrostriction effects in dielectric elastomer composites containing polarizable inclusions with different spatial distributions. Phys Chem Chem Phys 2015;17:32479-97.

53. AllahyarovE, Löwen H, Zhu L. Dipole correlation effects on the local field and the effective dielectric constant in composite dielectrics containing high-k inclusions. Phys Chem Chem Phys 2016;18: 19103-17.

54. Allahyarov E, Menzel AM, Zhu L, Löwen H. Magnetomechanical response of bilayered magnetic elastomers. Smart Mater Struct 2014;23:115004.

55. Menzel AM. Hydrodynamic description of elastic or viscoelastic composite materials: relative strains as macroscopic variables. Phys Rev E 2016;94:023003.

56. Attaran A, Brummund J, Wallmersperger T. Modeling and finite element simulation of the magnetomechanical behavior of ferrogels. J Magn Magn Mater 2017;431:188-91.

57. Attaran A, Brummund J, Wallmersperger T. Development of a continuum model for ferrogels. J Intell Mater Syst Struct 2017;28:1358-75.

58. Gebhart P, Wallmersperger T. A general framework for the modeling of porous ferrogels at finite strains. J Mech Phys Solid 2019;122:69-83.

59. Menzel AM. Bridging from particle to macroscopic scales in uniaxial magnetic gels. J Chem Phys 2014;141:194907.

60. Bohlius S, Brand HR, Pleiner H. Macroscopic dynamics of uniaxial magnetic gels. Phys RevE 2004; 70:061411.

61. Evans R. Density functional theory for inhomogeneous fluids I: simple fluids in equilibrium. In: Cichocki B, Napiórkowski M, Piasecki J, editors. Lecture notes 3rd Warsaw School of Statistical Physics. Warsaw: Warsaw University Press; 2010:43-85.

62. Löwen H. Density functional theory for inhomogeneous fluids II: statics, dynamics, and applications. In: Cichocki B, Napiórkowski M, Piasecki J, editors. Lecture notes 3rd Warsaw School of Statistical Physics. Warsaw: Warsaw University Press; 2010:87-121.

63. Cremer P, Heinen M, Menzel AM, Löwen H. A density functional approach to ferrogels. J Phys Condens Matter 2017;29:275102.

64. Goh S, Wittmann R, Menzel AM, Löwen H. Classical density functional theory for a two-dimensional isotropic ferrogel model with labeled particles. Phys Rev E 2019;100:012605.

65. Gundermann T, Cremer P, Löwen H, Menzel AM, Odenbach S. Statistical analysis of magnetically soft particles in magnetorheological elastomers. Smart Mater Struct 2017;26:045012.

66. Cremer P, Löwen H, Menzel AM. Tailoring superelasticity of soft magnetic materials. Appl Phys Lett 2015;107:171903.

67. Cremer $P$, Löwen H, Menzel AM. Superelastic stress-strain behavior in ferrogels with different types of magneto-elastic coupling. Phys Chem Chem Phys 2016;18:26670-90.

68. Menzel AM, Pleiner H, Brand HR. Response of prestretched nematic elastomers to external fields. Eur Phys J E 2009;30:371. 
69. Menzel AM, Pleiner H, Brand HR. On the nonlinear stress-strain behavior of nematic elastomers materials of two coupled preferred directions. J Appl Phys 2009;105:013503.

70. Schopphoven C, Birster K, Schweitzer R, Lux C, Huang S, Kästner M, et al. Elastic deformations in semi-dilute Ni nanorod/hydrogel composites. Arch Appl Mech 2019;89:119-32.

71. Biller AM, Stolbov OV, Raikher YL. Modeling of particle interactions in magnetorheological elastomers. J Appl Phys 2014;116:114904.

72. Pessot G, Weeber R, Holm C, Löwen H, Menzel AM. Towards a scale-bridging description of ferrogels and magnetic elastomers. J Phys Condens Matter 2015;27:325105.

73. Weeber R, Kantorovich S, Holm C. Ferrogels cross-linked by magnetic nanoparticles - deformation mechanisms in two and three dimensions studied by means of computer simulations. J Magn Magn Mater 2015;383:262-6.

74. Weeber R, Kantorovich S, Holm C. Ferrogels cross-linked by magnetic particles: field-driven deformation and elasticity studied using computer simulations. J Chem Phys 2015;143:154901.

75. Allahyarov E, Löwen H, Taylor PL. Simulation study of ion diffusion in charged nanopores with anchored terminal groups. Electrochim Acta 2017;242:73-85.

76. Wang H, Jacobi F, Waschke J, Hartmann L, Löwen H, Schmidt S. Elastic modulus dependence on the specific adhesion of hydrogels. Adv Funct Mater 2017;27:1702040.

77. Bechinger C, Di Leonardo R, Löwen H, Reichhardt C, Volpe G, Volpe G. Active particles in complex and crowded environments. Rev Mod Phys 2016;88:045006.

78. Babel S, Löwen H, Menzel AM. Dynamics of a linear magnetic "microswimmer molecule". EPL (Europhys Lett) 2016;113:58003.

Supplementary Material: The online version of this article offers supplementary material (https://doi. org/10.1515/psr-2019-0088). 